\title{
Solar Sail Propulsion for Interplanetary Cubesats
}

Les Johnson, Alex Sobey, and Kevin Sykes

NASA George C. Marshall Space Flight Center, Huntsville, AL 35812

les.johnson@nasa.gov

NASA is developing two small satellite missions as part of the Advanced Exploration Systems (AES) Program, both of which will use a solar sail to enable their scientific objectives. Solar sails use sunlight to propel vehicles through space by reflecting solar photons from a large, mirror-like sail made of a lightweight, highly reflective material. This continuous photon pressure provides propellantless thrust, allowing for very high $\Delta \mathrm{V}$ maneuvers on long-duration, deep space exploration. Since reflected light produces thrust, solar sails require no onboard propellant. Solar sail technology is rapidly maturing for space propulsion applications within NASA and around the world.

Lunar Flashlight, managed by JPL, will search for and map volatiles in permanently shadowed Lunar craters using a solar sail as a gigantic mirror to steer sunlight into the shaded craters. The Lunar Flashlight spacecraft will use the propulsive solar sail to maneuver into a lunar polar orbit. It will then use the same sail as a mirror to reflect $50 \mathrm{~kW}$ of sunlight down into shaded Polar Regions while an onboard spectrometer measures surface reflection and composition. The Lunar Flashlight uses a $6 \mathrm{U}$ cubesat architecture and will weigh less than 12 kilograms. The mission will demonstrate a low-cost capability to explore, locate and estimate the size and composition of ice deposits on the Moon.

The Near Earth Asteroid (NEA) Scout mission, managed by MSFC, will use the sail as primary propulsion allowing it to survey and image one or more NEA's of interest for possible future human exploration. Detections of NEAs are expected to grow in the near future, offering increasing target opportunities. Before NASA commits to sending people to a NEA, initial reconnaissance with comparatively inexpensive robotic precursors is necessary. Obtaining and analyzing relevant data about these bodies via robotic precursors before committing a crew to visit a NEA will significantly minimize crew and mission risk, as well as maximize exploration return potential. The NEA Scout spacecraft is nearly identical to the one being developed for Lunar Flashlight, with the science instrument package being the primary difference. The NEA Scout solar sail will provide the primary propulsion taking the $6 \mathrm{U}$ cubesat from near the Earth to its final asteroid destination. Both projects will use an $85 \mathrm{~m}^{2}$ solar sail developed by NASA MSFC.

The NEA Scout and Lunar Flashlight flight systems are based on a $6 \mathrm{U}$ cubesat form factor, with a stowed envelope slightly larger than $10 \times 20 \times 30 \mathrm{~cm}$ and a mass of less than $12 \mathrm{~kg}$. The flight systems follow a modular configuration, roughly divided into three sections stacked along the longest axis: the avionics module, the solar sail system, and a cold gas propulsion system. The avionics module accommodates a "stack" of typical cubesat-sized $(\sim 10 \times 10 \mathrm{~cm})$ printed circuit boards for the telecommunications, power distribution unit, command and data handling system (C\&DH), and a star tracker. This module also includes reaction wheels, batteries, and optical payload, and may be enclosed by additional shielding to manage the total ionizing dose on the most sensitive components. Situated close to the spacecraft center of mass between the cold gas and avionics module is the solar sail propulsion system. A cold gas 
propulsion system is situated below the solar sail and provides detumbling, initial impulsive maneuvers (required for lunar-assisted escape trajectories), and momentum management by desaturating the reaction wheels. Solar arrays are deployed in the plane of the solar sail, which also serves as the mounting location for a planar patch antenna array.

The spacecraft will use a deep-space attitude control architecture that responds to the unique attitude control considerations imposed by the solar sail while leveraging commercially available hardware. Detumbling and safing maneuvers rely on an inertial measurement unit, sun sensors, and the system actuators. Momentum management is primarily accomplished by spinning the solar sail slowly about the norm of the sail to average out - over the course of hours - the momentum build up due to any small but persistent offset in the solar sail's center of pressure of the system and the center of mass. In order to avoid spin-stabilizing the system to enable the system to stop spinning without expending cold gas, a large reaction wheel is used to absorb the momentum associated with the spinning sail. Smaller reaction wheels are used to maneuver and point the spacecraft based on the current slewing/pointing commands. When these wheels saturate, the system uses the cold gas to dump the momentum in the system. Pointing and slewing is accomplished primarily with a star tracker and gyro for attitude knowledge, and the smaller reaction wheels for attitude control. The inclusion of a large flexible body that increases the system's sensitivity to solar radiation torques clearly impacts the Attitude Control System (ACS) pointing error budget and thus the subsystem design.

Solar sail propulsion was demonstrated in space by the Japanese Aerospace Exploration Agency (JAXA) with the successful flight of their Interplanetary Kite-craft Accelerated by Radiation of the Sun (IKAROS) in 2010. IKAROS was the first of several sail demonstration missions to actually fly in space. NASA invested in the technology in the late 1990's, maturing them to Technology Development Level 6. NASA MSFC collaborated with the NASA Ames Research Center to develop the NanoSail-D, a subscale aerodynamic drag sail system designed for small spacecraft applications. The NanoSail-D flew aboard the Fast Affordable Science and Technology SATellite (FASTSAT) in 2010, and began its mission after it was ejected from the FASTSAT into Earth orbit, where it remained for several weeks before deorbiting as planned.

The University of Surrey is developing multiple cubesat-class solar and drag sail spacecraft, including CubeSail, Deorbit Sail and InflateSail. Each will have a total mass of around $3 \mathrm{~kg}$ and will deploy $16 \mathrm{~m}^{2}$ sails in low Earth orbit. At the same time, The Planetary Society is developing its own cubesat-scale solar sails that will fly on the LightSail-A and LightSail-B missions.

The solar sail for NEA Scout and Lunar Flashlight will be based on the technology developed and flown by the NASA NanoSail-D and The Planetary Society's Lightsail-A. Four $7 \mathrm{~m}$ stainless steel booms wrapped on two spools (two overlapping booms per spool) will be motor deployed and pull the sail from its stowed volume. The sail material will be $3 \mathrm{~m} \mathrm{CP1}$, an aluminized polyimide that was extensively tested for solar sail applications. The sail will spooled rather than Z-folded. 
As the technology matures, solar sails will increasingly be used to enable science and exploration missions that are currently impossible or prohibitively expensive using traditional chemical and electric rockets. This paper will summarize the status of solar sail technology, current and planned sail missions. 